\title{
Combined Treatment of Photobiomodulation and Arginine on Chronic Wound Healing in an Animal Model
}

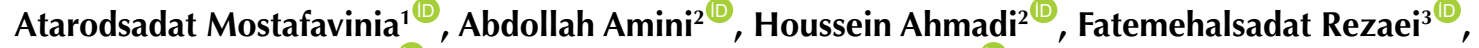 \\ Seyed Kamran Ghoreishi ${ }^{\circledR}$, Sufan Chien ${ }^{5 *}$, Mohammad Bayat ${ }^{2,5^{*}}$ \\ ${ }^{1}$ Department of Anatomy, Faculty of Medicine, Tehran Medical Sciences, Islamic Azad University, Tehran, Iran \\ ${ }^{2}$ Department of Biology and Anatomical Sciences, School of Medicine, Shahid Beheshti University of Medical \\ Sciences (SBMU), Tehran, Iran \\ ${ }^{3}$ University of Kentucky College of Pharmacy 789 South Limestone Lexington, Kentucky 40536, USA \\ ${ }^{4}$ Department of Statistics, University of Qom, Qom, Iran \\ ${ }^{5}$ Price Institute of Surgical Research, University of Louisville, and Noveratech LLC of Louisville, Louisville, \\ Kentucky, USA
}

\section{*Correspondence to Mohammad Bayat, \\ Email: bayat_m@yahoo.com Sufan Chien, \\ Email: sufan.chien@louisville. edu}

Received: August 2, 2020 Accepted: March 6, 202 Published online July 24, 2021

\begin{abstract}
Introduction: Herein, the individual and combined effects of photobiomodulation (PBM) and arginine (ARG) on the wound healing course of an experimental model of a slow healing wound (ulcer) in rats were assessed.

Methods: A total of 108 male rats were divided into 6 groups: control; lower energy density (low)-PBM; arginine ointment (ARG); low-PBM+ARG; high energy density (high)-PBM; and high$\mathrm{PBM}+\mathrm{ARG}$. In each rat, one ischemic wound in the center of a bipedicle flap and one non-ischemic wound out of the flap were created. Both wounds were treated in the experimental groups. Microbial growth, wound area, and wound strength were assessed on days $0,5,10,15$, and 20 after wound infliction.

Results: All non-ischemic wounds closed before day 15. High-PBM+ARG and ARG significantly increased wound closure rates compared to the control group (LSD test, $P=0.000$, and $P=0.001$, respectively) on day 10 . All slow healing wounds were open on day 15 but closed completely before day 20. Low-PBM+ARG and high-PBM significantly increased wound strength (stress high load, SHL) on day 10 compared to the control group (LSD test, $P=0.001$, and $P=0.000$, respectively). ARG, high-PBM, and low-PBM+ARG significantly increased wound closure rates on day 15 relative to the control group (LSD test, $P=0.000, P=0.000$, and $P=0.001$, respectively).

Conclusion: High-PBM and low-PBM+ARG have biostimulatory and antibacterial effects on slowhealing wounds, which were shown by significant increases in wound closure rates, wound strength, and inhibition of Staphylococcus aureus growth.

Keywords: Photobiomodulation; Arginine; Chronic wound; Wound closure rate; Tensiometry.
\end{abstract}

\section{Introduction}

The inhibition of typical healing results in the development of chronic wounds (ulcers). Ischemia is a highly important cause of chronic wounds that are not repaired in a reasonable length of time. ${ }^{1}$ Ischemia is attributed to an inadequate arterial blood flow, which results in ischemic ulcers. ${ }^{2}$ It diminishes the number of neutrophils and macrophages, ${ }^{3}$ predisposes ulcers to infection, inflammation, and necrosis, causes damage to myofibroblast progression in granulation tissue, ${ }^{4}$ and is a vital component for interrupted healing in several illnesses. ${ }^{5}$ Chronic wounds are a medical concern because they impede the ability of the skin to repair itself. ${ }^{6}$ In the United States, chronic wounds affect approximately 6.5 million people, and more than $\$ 25$ billion are spent annually for the management of chronic wounds. ${ }^{7}$

Arginine (ARG) is an important non-essential amino acid reported to improve wound strength and collagen release in wounds. ${ }^{8}$ It is the nitrogenous precursor for nitric oxide (NO) synthesis, and it controls important 
metabolic pathways. ${ }^{9}$ According to research, ARG in an ischemic flap model has been shown to stimulate flap viability. ${ }^{10}$

Photobiomodulation (PBM) is a non-warming light and a recommended treatment for a number of illnesses due to its comfort, effectiveness, and decreased cost compared to current treatment methods. ${ }^{11}$ It decreases pain and inflammation, enhances injury repair, and prevents cell and tissue damage. ${ }^{12}$ PBM is reported to enhance new blood vessel formation ${ }^{13}$ and flap survival, ${ }^{14}$ increase wound bed volume, and create an anti-inflammatory effect on ischemic tissue. ${ }^{15}$

In this study, it was hypothesized that a mixture of beneficial biomediators (PBM and ARG) can be used to accelerate the healing course in an ischemic and slow healing wound model because of its antioxidant and antiinflammatory activities and triggering effects. Accordingly, we previously reported that a combination of PBM and ARG induced anti-inflammatory and angiogenic activities and hastened the wound healing process in an excisional wound model in rats. ${ }^{16}$ The current study assessed the effects of PBM and ARG, individually and together, on wound closure rates, microbial flora, and wound strength in a rat model of ischemic and slow-healing ulcer.

\section{Materials and Methods Animals and Study Design}

A total of 108 adult male Wistar rats were housed individually in a standard animal home with ad libitum access to rat food pellets and water. The rats were randomly divided into 6 groups $(\mathrm{G})$ of 6 rats each as follows: control (G1); lower energy density (low)-PBM (G2); arginine ointment (G3); low-PBM+ARG (G4); high energy density (high)-PBM (G5); and high-PBM+ARG (G6). First, a bipedicle skin flap was created on the back area of each rat, followed by an excisional wound in the flap. A donut-shaped silicone skin holder was fixed around each wound. ${ }^{17,18}$ A full-thickness, non-ischemic wound distal to the flap served as the internal, non-ischemic wound (Figure 1). On days 0, 5, 10, 15, and 20, bacterial evaluations, wound closure rate assessments, and wound strength tests were performed. Day 5 was considered the inflammation phase, day 10 was the proliferation phase, day 15 was the early remodeling phase, and day 20 was the late remodeling phase in the current ischemic and delayed wound healing course.

\section{Surgery}

A dorsal, bipedicle skin flap $(10 \times 3.5 \mathrm{~cm})$ was induced deep into the skin muscle under aseptic conditions and general anesthesia. The flaps were sutured to adjacent skin edges. One 12-mm, full-thickness excisional ulcer that included the skin muscle was produced in the midpoint region of the flap by a biopsy punch. Figure 1 shows a donut-shaped silicone skin holder which was fixed around

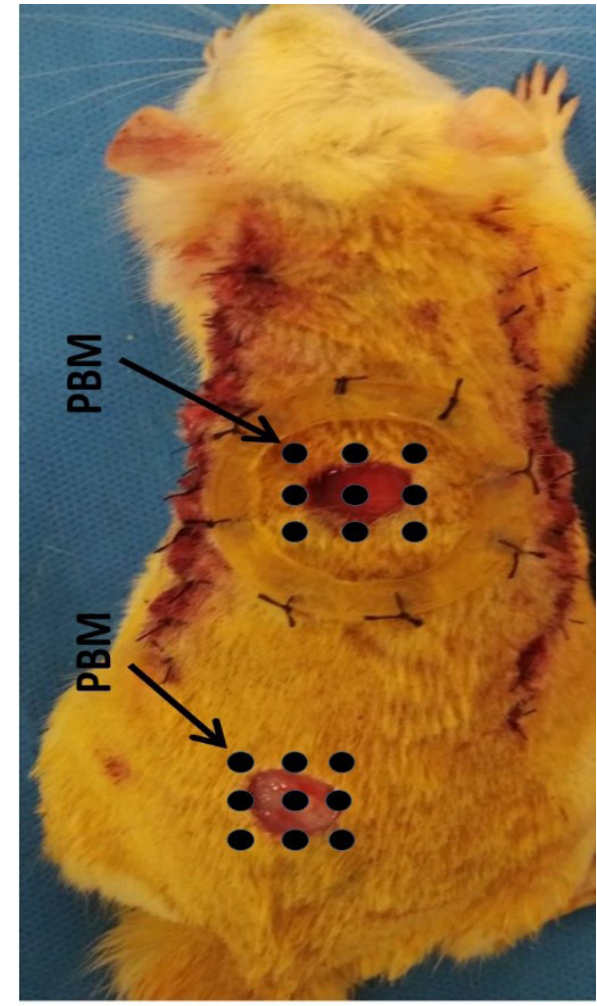

Figure 1. A Photo of the Ischemic and Slow Healing Wound Model With its Control Non-ischemic Wound and Photobiomodulation (PBM) Points.

each skin defect with a 04 silk suture. ${ }^{18}$ A full-thickness, non-ischemic wound distal to the flap served as the internal, non-ischemic wound. The skin of the back was shaved and topically disinfected with povidone-iodine. A sterile surgical set was used. Ceftriaxone $(50 \mathrm{mg} / \mathrm{kg})$ as an antibiotic medicine was injected immediately, 24 hours, and 48 hours after surgery.

\section{Administration of PBM and ARG Ointment}

Both slow-healing ischemic and non-ischemic wounds were treated. PBM was started immediately after surgery. Nine shootings of PBM were performed over 9 defined regions of the ischemic wound and nearby skin (Figure 1) according to the protocol given below. A MUSTANG 2000, LO7 probe (Technica Co., Russia) with the following specifications was used: $1.08 \mathrm{~mW} / \mathrm{cm}^{2}$ power density, 75 $\mathrm{W}$ peak power output, $1.08 \mathrm{~mW}$ average power, $1 \mathrm{~cm}^{2}$ spot size, $80 \mathrm{~Hz}$ pulse rate, and $890 \mathrm{~nm}$ infra-red wavelength. The pulse duration was $180 \mathrm{~ns}$ with energy densities of 0.2 $\mathrm{J} / \mathrm{cm}^{2}(200 \mathrm{~s})$ and $0.32 \mathrm{~J} / \mathrm{cm}^{2}$ (300 s). ${ }^{16}$

Arginine powder (Sigma-Aldrich, USA) (2 g) was dissolved in water, and $98 \mathrm{~g}$ of Eucerin was added under sterile conditions to create the ARG ointment. ${ }^{16}$ Both PBM and approximately $0.04 \mathrm{~g}$ of the ARG ointment were applied to the wounds of groups G4 and G6. Group G3 received only the ARG ointment, and groups G2 and G5 received only PBM. PBM and ARG were administered 
once daily, 6 days per week, for a maximum of 20 days.

\section{Wound Closure Evaluation}

Photos of the wounds were taken on days $0,5,10,15$, and 20. Wound areas were calculated by NIH ImageJ (USA). ${ }^{16}$ Wound closure rates were calculated and compared in the study groups and at each time point. The wound closure rate was calculated according to the following formula:

Rate of wound closure $=\frac{\text { surface area of day } \times\left(\mathrm{mm}^{2}\right)}{\text { surface area at day zero }\left(\mathrm{mm}^{2}\right)} \times 100$

\section{Microbiological Analyses}

Swabs were taken from the wounds of the study groups on days 0,10 , and 20 to detect Pseudomonas aeruginosa as the gram-negative bacteria and Staphylococcus aureus as the gram-positive bacteria, using Müller-Hinton Agar (MHA), a nutrient medium for both named bacteria. The samples were cultured in the Müller-Hinton culture medium for 24 hours at $37^{\circ} \mathrm{C}$. The Mannitol salt culture medium was then used to distinguish between $S$. aureus and S. epidermidis. Finally, the microbes in each sample were counted as colony-forming units (CFUs). ${ }^{19}$

\section{Wound Strength Examination}

Uniform samples $(5 \times 50 \mathrm{~mm}$ bands $)$ were taken from each wound on the euthanized rats on days 5,10 , and 20 and fixed in a material testing machine. The deformation rate was $10 \mathrm{~mm} / \mathrm{min}$. The load-deformation curve was used to calculate the bending stiffness (BS) and stress high load (SHL, N/mm²) of the samples. ${ }^{20}$

\section{Statistical Analyses}

The data are presented as mean \pm standard deviation (SD). The $t$ test, one-way analysis of variance (ANOVA), two-way ANOVA, and the least significant difference (LSD) tests were used for statistical analyses. A $P$ value $<0.05$ was considered statistically significant.

\section{Results \\ Clinical Observations}

No wound exudate or other clinical complications were observed during the experiment. There were significant changes in the rats' weights on day 20 compared to day 0 in some of the study groups (Table 1).

\section{Wound Closure Rate}

Figure 2 shows the one-way ANOVA for the wound closure rates on days 5,10 , and 15 .

In the ischemic + ring group, there was no significant difference in wound closure rates among the studied groups on day 5 . All wounds were closed by day 20 .

LSD analysis showed that on day 10, the ARG and control groups had a significantly decreased wound closure rate compared to the low-PBM $[P=0.000$
Table 1. Study Rats'Weights on Days 0 and 20

\begin{tabular}{lcc}
\hline Groups & $\begin{array}{c}\text { Weight (g) } \\
\text { Day 0 }\end{array}$ & $\begin{array}{c}\text { Weight (g) } \\
\text { Day 20 }\end{array}$ \\
\hline Control (G1) & $222.00 \pm 7.04$ & $244.50 \pm 11.43^{*}$ \\
Low-PBM (G2) & $248.50 \pm 12.49$ & $247.50 \pm 13.97$ \\
Arginine (G3) & $264.60 \pm 11.28$ & $292.20 \pm 12.68^{* *}$ \\
Low-PBM+ARG (G4) & $251.17 \pm 8.59$ & $251.66 \pm 7.53$ \\
High-PBM (G5) & $239.00 \pm 6.00$ & $246.66 \pm 10.33^{*}$ \\
High-PBM+ARG (G6) & $243.00 \pm 11.48$ & $235.33 \pm 14.63$ \\
\hline
\end{tabular}

Study groups: Control (G1), low-PBM (G2), arginine (G3), lowPBM+ARG (G4), high-PBM (G5), high-PBM+ARG (G6); PBM, photobiomodulation; t-test; ${ }^{*} P<0.05, * * P<0.01$

(ARG), $P=0.005$ (control)], low-PBM+ARG $[P=0.000$ (ARG), $\quad P=0.005$ (control) $], \quad$ high-PBM $[P=0.000$ (ARG), $P=0.003$ (control)], and high-PBM+ARG (both, $P=0.000$ ) groups. On day 15 , the ARG, low-PBM+ARG, and high-PBM groups had significantly increased wound closure rates compared to the high-PBM (all, $P=0.000$ ), low-PBM ( $P=0.001, P=0.004, P=0.001)$, and control $(P=0.000, P=0.001, P=0.000)$ groups, as presented in Figure 2A.

In the rats with non-ischemic wounds without the ring, all wounds were closed on day 15 . On day 5 , significantly higher wound closure rates were observed in the low-

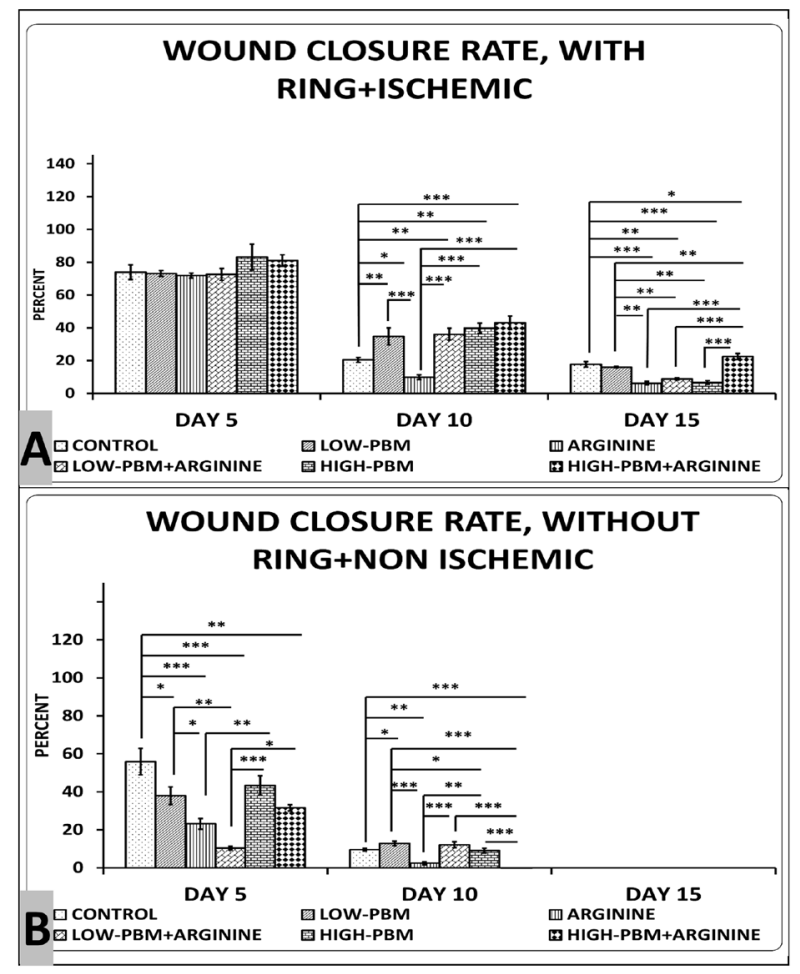

Figure 2. Mean \pm Standard Deviation of the Wound Closure Rate in the Ischemic + Ring (A) Group, and Wound Closure Rate of the Non-ischemic Without the ring (B) group on days 5, 10, and 15 as Compared by ANOVA and Least Significant Difference. ${ }^{*} P<0.05$, ${ }^{* *} P<0.01$, and $* * * P<0.001 ; \mathrm{PBM}$, photobiomodulation; ischemic + ring $=$ slow healing. 
PBM+ARG $\quad(P=0.000), \quad$ ARG $\quad(P=0.000), \quad$ low-PBM $(P=0.012)$, and high-PBM+ARG $(P=0.006)$ groups compared to the control group. The low-PBM+ARG group had a significantly higher wound closure rate than the low-PBM $(P=0.001)$, high-PBM $(P=0.000)$, and high-PBM+ARG $(P=0.013)$ groups. On day 10 , wounds in the high-PBM group were closed, and significantly higher wound closure rates were observed in the high$\mathrm{PBM}+\mathrm{ARG}$ and ARG groups compared to the highPBM ( $P=0.000, P=0.001), P B M+A R G$, low-PBM (all $P=0.000)$, and control $(P=0.000, P=0.001)$ groups (Figure 2B).

Two-way ANOVA revealed significant reductions in wound surface area in each of the ischemic + ring groups during the treatment time point. A similar result was also observed for non-ischemic wounds without the ring. In both cases, effectiveness in the wound healing course was higher in the PBM treatment groups compared to the control group. During the early days of the wound healing course, the PBM+ARG treatment group showed more rapid wound healing as demonstrated in Table 2 and Figure 3.

\section{Microbiological Analysis}

$P$. aeruginosa was not observed in the cultured samples. Due to tremendous differences in CFUs for $S$. aureus in the study groups, statistical analysis was not possible. Table 3 shows the mean \pm SD for $S$. aureus and S. epidermidis CFUs. Meaningful changes in S. epidermidis were found between the treatment and control groups as revealed in Table 3.

\section{Wound Strength Test for the Ulcer (Ischemic + Ring) Groups}

Figure 4 shows the results of the wound strength test.

On day 5 , significantly increased bending stiffness (BS) was observed in the high-PBM+ARG $(P=0.001)$, highPBM $(P=0.003)$, and low-PBM+ARG $(P=0.049)$ groups compared to the ARG group. On day 10, the BS values for the high-PBM+ARG $(P=0.000)$, low-PBM $(P=0.000)$, low-PBM+ARG $(P=0.001), \mathrm{PBM}(P=0.007)$, and ARG $(P=0.012)$ groups significantly increased compared to the control group. The high-PBM+ARG $(P=0.000)$

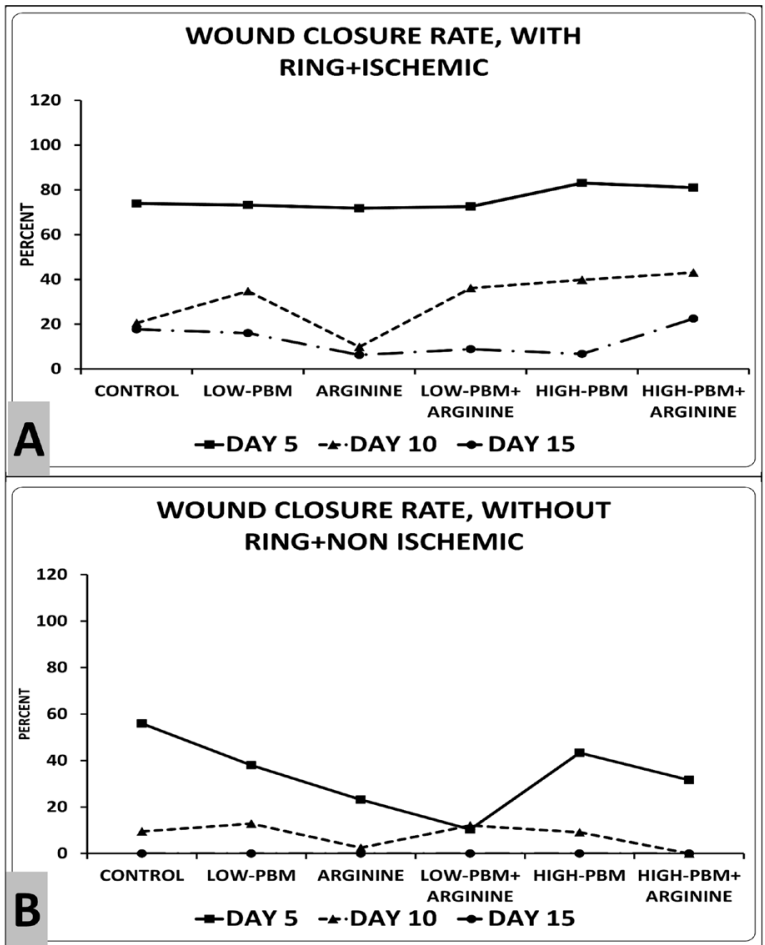

Figure 3. Two-way ANOVA for ischemic wound closure rate with the ring $(A)$ showed a significant reduction in the wound surface area in all groups during the treatment time point. A similar result was observed for non-ischemic wounds without the ring (B). In both cases, the photobiomodulation (PBM) groups were more effective in wound healing compared to the control group. In the early days of the wound healing process, more rapid wound healing was observed in the photobiomodulation + arginine treatment groups.

and low-PBM $(P=0.001)$ groups had significantly increased BS compared to the ARG group. On day 20, BS significantly increased in the ARG group compared to the low-PBM+ARG $(P=0.003)$, high-PBM $(P=0.049)$, high$\mathrm{PBM}+\mathrm{ARG}(P=0.013)$, and control $(P=0.012)$ groups, as demonstrated in Figure 4A.

Figure $4 \mathrm{~B}$ demonstrates that on day 5 , the high-PBM and high-PBM+ARG groups had a significantly increased SHL compared to the ARG $(P=0.014, P=0.003)$, lowPBM $(P=0.003, \quad P=0.023)$, and control $(P=0.002$, $P=0.014)$ groups. On day 10 , the high-PBM+ARG $(P=0.000), \quad$ low-PBM $\quad(P=0.000), \quad$ low-PBM+ARG

Table 2. Two-Way ANOVA of Wound Closure Rates in Ischemic and Non-ischemic Wounds Following the Treatment With the Study Groups on Days 5, 10, and 15

\begin{tabular}{|c|c|c|c|c|}
\hline Parameter & Source & F-Statistic & $\begin{array}{c}\text { Significance } \\
\text { Level }\end{array}$ & Result \\
\hline \multirow{3}{*}{ Ischemic wound } & Group & 9.6 & 0.000 & \multirow{3}{*}{ Significant differences between days among groups } \\
\hline & Day & 503 & 0.000 & \\
\hline & Group $\times$ Day & 4.3 & 0.001 & \\
\hline \multirow{3}{*}{ Non-ischemic wound } & Group & 8.8 & 0.000 & \multirow{3}{*}{ Significant differences between days among groups } \\
\hline & Day & 173 & 0.000 & \\
\hline & Group $\times$ Day & 8.5 & 0.000 & \\
\hline
\end{tabular}


Table 3. Mean \pm SD Colony-Forming Units for Staphylococcus aureus and Staphylococcus epidermidis in the Studied Groups on Days 0, 10 , and 20

\begin{tabular}{|c|c|c|c|}
\hline Day & Bacteria Group & S. epidermidis & S. aureus \\
\hline \multirow{6}{*}{0} & Control & $83.33 \pm 29.43$ & $0 \pm 0$ \\
\hline & Low-PBM & $166.66 \pm 30.55$ & $0 \pm 0$ \\
\hline & Arginine & $353.33 \pm 128.58^{* *}$ & $0 \pm 0$ \\
\hline & Low-PBM+ARG & $165.00 \pm 49.49$ & $0 \pm 0$ \\
\hline & High-PBM & $395.00 \pm 186.45^{* *}$ & $0 \pm 0$ \\
\hline & High-PBM+ARG & $216.00 \pm 141.70$ & $0 \pm 0$ \\
\hline \multirow{6}{*}{10} & Control & $96788.00 \pm 4529.31$ & $98021.80 \pm 1920.13$ \\
\hline & Low-PBM & $180.00 \pm 28.28^{* * *}$ & $4800.00 \pm 380.21$ \\
\hline & Arginine & $370.00 \pm 127.27^{* * *}$ & $3640.00 \pm 56.56$ \\
\hline & Low-PBM+ARG & $370.00 \pm 127.27^{* * *}$ & $0 \pm 0$ \\
\hline & High-PBM & $1163.33 \pm 352.91^{* * *}$ & $0 \pm 0$ \\
\hline & High-PBM+ARG & $11333.33 \pm 2753.78^{* * *}$ & $98566.66 \pm 3510.93$ \\
\hline \multirow{6}{*}{20} & Control & $97390.00 \pm 4231.00$ & $0 \pm 0$ \\
\hline & Low-PBM & $92893.33 \pm 2591.16$ & $0 \pm 0$ \\
\hline & Arginine & $713.33 \pm 70.23^{* * *}$ & $0 \pm 0$ \\
\hline & Low-PBM+ARG & $95200.00 \pm 6788.22$ & $0 \pm 0$ \\
\hline & High-PBM & $843.33 \pm 171.30^{* * *}$ & $0 \pm 0$ \\
\hline & High-PBM+ARG & $95000.00 \pm 5477.22$ & $0 \pm 0$ \\
\hline
\end{tabular}

Due to tremendous differences in the colony-forming units for $S$. aureus in the studied groups, we could not perform statistical analyses. ${ }^{* *} P<0.01$ and ${ }^{* * *} P<0.001$ : Significant differences in $S$. epidermidis between the treatment and control groups; PBM, photobiomodulation.

$(P=0.001)$, and high-PBM $(P=0.007)$ treatments had a significantly increased SHL compared to the control group. The high-PBM+ARG $(P=0.000)$, low-PBM $(P=0.001)$, and low-PBM+ARG $(P=0.019)$ groups had a significantly increased SHL compared to the ARG group. On day 20, MF significantly increased in the ARG group compared to the low-PBM+ARG and high-PBM+ARG groups ( $P=0.002$ and $P=0.018$, respectively).

Two-way ANOVA for the SHL showed significant differences among the groups on different days. For almost all groups, the SHL values on day 5 were dominated by the corresponding values on days 10 and 20. In terms of SHL, both the low-PBM and high-PBM+ARG groups had a relatively good performance in wound healing. The assessments of BS showed that the low-PBM, low$\mathrm{PBM}+\mathrm{ARG}$, and high-PBM+ARG groups had the highest values for these two factors (different groups and different time points). Moreover, for all groups, the BS values on day 5 were dominated by the corresponding values on days 10 and 20.

\section{Discussion}

The current results showed that the high-PBM+ARG and ARG groups had significantly increased wound closure rates compared to the other PBM treatment groups and the control group on day 10.

Growth of $S$. aureus was observed in the high$\mathrm{PBM}+\mathrm{ARG}(98566 \pm 3550 \mathrm{CFU})$, control (98021 \pm 1920
CFU), low-PBM (4800 $\pm 380 \mathrm{CFU})$, and ARG (3640 \pm 56 $\mathrm{CFU})$ groups on day 10 .

Animal models are important for information gathering ${ }^{21}$ and the study of human diseases in order to direct laboratory results into medical applications. ${ }^{22}$ Localized tissue ischemia is an important issue in the progress and prognosis of chronic wounds. ${ }^{23}$ Rats have a skin muscle that has been reported to share in skin repair by permitting wound closure. ${ }^{24}$ According to Gould et al, their ischemic wound model is unfailing, inexpensive, and simple, and prevents wound closure by the skin muscle. ${ }^{25}$ We generated this model in rats by creating a bipedicle skin flap deep into the skin muscle, then inserting a silicone sheet to inhibit wound closure. Our pilot study showed that this model was successful in approximately $50 \%$ of the animals, with an increased risk of microbial infection. Accordingly, other studies used a narrower bipedicle skin flap without a silicone sheet. This model had many of the biological features observed in chronic wounds in humans. ${ }^{26,27}$ Recently, Fukui et al and Ren et al implanted a silicone circle around wounds in rodents to prevent wound closure by the skin muscle, as shown in Figure 1. This method was less invasive and more practical than the insertion of a large silicone sheath under the entire bipedicle skin graft. It could effectively reduce wound closure and allow for wound healing mainly through reepithelialization and granulation tissue formation, with a decreased risk of microbial infection. ${ }^{28}$ Therefore, this 


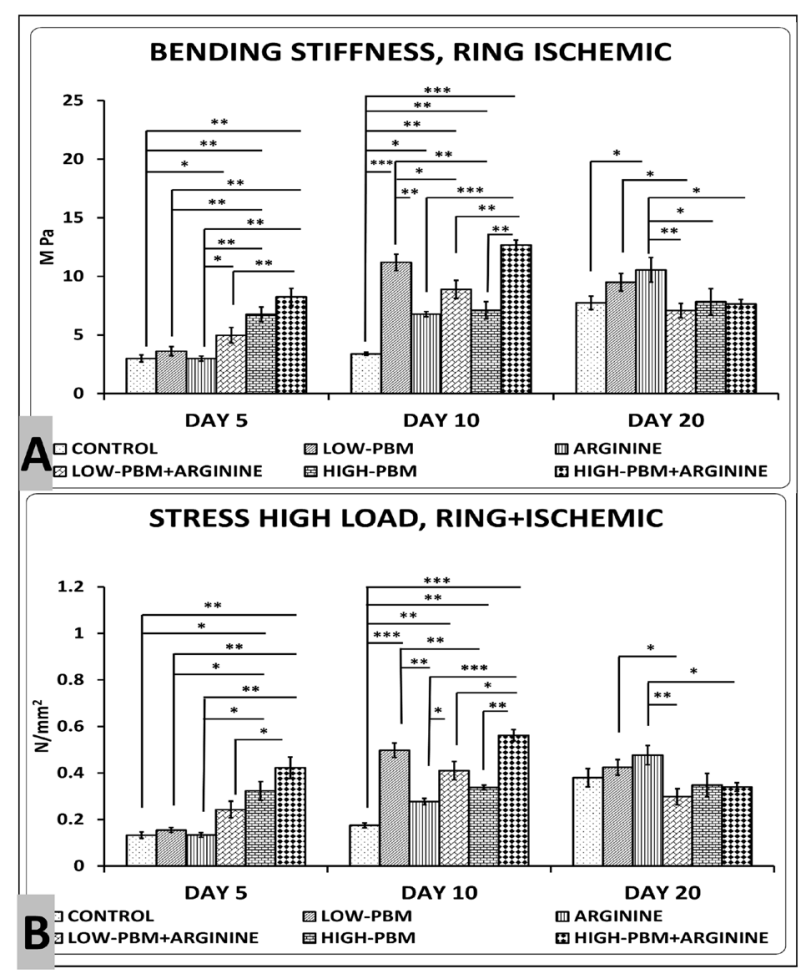

Figure 4. Mean \pm Standard Deviation of Bending Stiffness (A) and Stress High Load (B) for the Ischemic + Ring Groups on Days 5, 10, and 15, Compared by One-Way ANOVA and least significant difference. ${ }^{*} P<0.05,{ }^{*} P<0.01$, and ${ }^{* * *} P<0.001 ; \mathrm{PBM}$, photobiomodulation; ischemic + ring $=$ slow healing.

method might be a better choice for the assessment of molecular and cellular actions within the chronic ulcer repair course. It also makes it easier to assess the effects on ischemic ulcer repair by external biostimulator agents. ${ }^{28,29}$

Chronic wounds consist of diabetic feet, venous legs, and pressure ulcers. The difficulty in healing such wounds presents a challenge for medical personnel and results in increased expenditures worldwide. Overcoming the factors that contribute to delayed repair is a key component in wound care and a main task in healing chronic ulcers. ${ }^{30}$

Collagens are vital components of all stages of skin injury repair. Collagens give the skin its integrity and strength and play a particularly important role in the proliferative and remodeling stages of skin injury repair. ${ }^{31}$ Wound strength and wound closure rates are the best measures to use in assessing surgical wound healing in humans. ${ }^{32,33}$ The current study demonstrated that treatment with PBM has beneficial effects on wound strength properties and wound closure rates for the repair of an ischemic and slow healing ulcer model in rats. PBM was successfully used to accelerate skin injury repair and positively impact inflammatory reactions. ${ }^{34}$ It would be extremely valuable for people who are needle-phobic or unable to tolerate non-steroidal anti-inflammatory drugs. ${ }^{35}$

Based on the principles of physics, skin wounds appear to be the finest matched wounds for PBM. Numerous experiments have shown the efficacy of PBM treatment for ischemic skin wounds. Recently, Kouhkheil et al and Fridoni et al reported the positive impacts of PBM on improving wound strength, microbial flora, fibroblasts and inflammatory cells, and molecules and growth factors in an infected and diabetic wound model in rats. ${ }^{36,37}$ Ruh et al reported that PBM (InGaAIP, $100 \mathrm{~mW}, 660 \mathrm{~nm}, 2$ $\mathrm{J} / \mathrm{cm}^{2}$ ) administered once per day improved the gross appearance of an ischemic pressure sore, increased vascular endothelial growth factor (VEGF), transformed growth factor- $\beta$ (TGF- $\beta$ ), and reduced tumor necrosis factoralpha (TNF- $\alpha) .{ }^{15}$ The beneficial results of PBM depend on the PBM protocol and require more consideration. The enhancement of flap viability, increased blood vasculature and cell counts in the proliferative phase of skin injury repair, and inhibited free-radicals have been described in some experiments with PBM (470 nm, 629 $\mathrm{nm}, 632.8 \mathrm{~nm}, 660 \mathrm{~nm}, 670 \mathrm{~nm}, 780 \mathrm{~nm}, 830 \mathrm{~nm}$, and $\left.2.9 \mathrm{~J} / \mathrm{cm}^{2}, 3 \mathrm{~J} / \mathrm{cm}^{2}, 36 \mathrm{~J} / \mathrm{cm}^{2}\right) .{ }^{38-41}$ Other experiments that have used different PBM protocols $(660 \mathrm{~nm}, 670 \mathrm{~nm}$, and $\left.780 \mathrm{~nm} ; 30,40,100 \mathrm{~J} / \mathrm{cm}^{2}\right)^{13,42-44}$ have reported decreased flap survival. Interestingly, Cury et al. used the same PBM protocol $\left(660\right.$ and $780 \mathrm{~nm}, 30$ and $40 \mathrm{~J} / \mathrm{cm}^{2}$ ) and reported two different results. In their initial experiment, they observed that PBM did not increase flap survival in rats. ${ }^{42}$ In their most recent experiment, however, they reported that PBM could modulate VEGF secretion and MMP-2 activity in a dose-dependent manner and consequently improved skin flap healing. ${ }^{41}$

Recently, Silva et al reported that ARG in a modified McFarlane ischemic flap model promoted flap survival by significantly decreasing malondialdehyde and $\mathrm{NO}_{2}$ and significantly increased non-protein thiol concentrations. They concluded that ARG moderated antilipoperoxidative action along with intense antioxidant activity in skin flaps in rats. ${ }^{10}$

Figure 2 shows the synergistic effects observed after the treatment with ARG and high-PBM on wound closure rates on day 15 in a non-ischemic, non-delayed ulcer model. This finding agreed with recent studies. Alexander et al, Farreras et al, and Okamoto et al reported that both ARG and fish oils have independent benefits, but a combination of the two appeared to be substantially more effective. ${ }^{45-47}$ Farreras et al and Okamoto et al reported that enteral nutrition with both ARG and omega 3 fatty acids improved surgical wound healing in patients. ${ }^{46,47}$

The current study determined that in the ischemic and slow healing ulcer model, PBM significantly increased wound strength properties during the proliferative phase compared to the control and ARG groups. High-PBM, low$\mathrm{PBM}+\mathrm{ARG}$, and ARG treatments significantly increased wound closure rates compared to the control group in the early phase of remodeling. The wound closure rate in rats with wounds treated with ARG was statistically better during the proliferative phase compared to the other 
groups. In terms of microbial flora, treatment with highPBM inhibited S. aureus growth in the ischemic and slow healing wounds in the proliferation phase. Evaluations of wound closure in the non-delayed, non-ischemic wound model showed that the high-PBM+ARG and ARG groups had significantly increased wound closure rates compared to the other PBM treatments and the control group during the proliferation phase.

It should be noted that $P$. aeruginosa as the gramnegative bacteria and $S$. aureus as the gram-positive bacteria are the most common as well as most dangerous microbes at the wound site to be examined. ${ }^{48,49}$ However, $P$. aeruginosa was not observed in the cultured samples of the current study.

\section{Conclusion}

The slow healing wound model in rats in the current study was established by comparing its wound closure rate with that of non-ischemic wounds that showed an approximately five-day delay in wound closure. According to the results, the biostimulatory and antibacterial effects of high-PBM and low-PBM+ARG on ulcers were demonstrated by significant increases in wound closure rates and wound strength and the inhibition of $S$. aureus growth.

The cellular and molecular mechanisms pertaining to the effects of PBM and ARG on the slow healing and ischemic wound model in rats should be determined by further investigations.

\section{Ethical Considerations}

The IRB of the National Institute for Medical Research Development (NIMAD), Tehran, Iran (file no: IR.NIMAD. REC.1397.256) approved all the experiments.

\section{Conflict of Interests}

The authors declare they have no conflicts of interest.

\section{Acknowledgment}

Research reported in this publication was partially supported by Elite Researcher Grant Committee under award number [971122] from the National Institutes for Medical Research Development (NIMAD), Tehran, Iran.

\section{References}

1. Schäffer M, Witte $M$, Becker H-D. Models to study ischemia in chronic wounds. Int J Low Extrem Wounds. 2002;1(2):104-11. doi: 10.1177/1534734602001002005.

2. Hirsch AT, Haskal ZJ, Hertzer NR, Bakal CW, Creager MA, Halperin JL, et al. ACC/AHA 2005 practice guidelines for the management of patients with peripheral arterial disease (lower extremity, renal, mesenteric, and abdominal aortic): A collaborative report from the american association for vascular surgery/society for vascular surgery,* Society for Cardiovascular Angiography and Interventions, Society for Vascular Medicine and Biology, Society of Interventional Radiology, and the ACC/AHA Task Force on Practice Guidelines (Writing Committee to Develop Guidelines for the Management of Patients With Peripheral Arterial Disease): Endorsed by the American Association of Cardiovascular and Pulmonary Rehabilitation; National Heart, Lung, and Blood Institute; Society for Vascular Nursing; TransAtlantic InterSociety Consensus; and Vascular Disease Foundation. Circulation. 2006;113(11):e463-e654. doi: 10.1161/ CIRCULATIONAHA.106.174526.

3. Egners A, Erdem M, Cramer T. The response of macrophages and neutrophils to hypoxia in the context of cancer and other inflammatory diseases. Mediators Inflamm. 2016;2016. doi: 10.1155/2016/2053646.

4. Alizadeh N, Pepper MS, Modarressi A, Alfo K, Schlaudraff $\mathrm{K}$, Montandon D, et al. Persistent ischemia impairs myofibroblast development in wound granulation tissue: a new model of delayed wound healing. Wound Repair Regen. 2007;15(6):809-16. doi: 10.1111/j.1524-475X.2007.00312.x

5. Sisco M, Mustoe TA. Animal models of ischemic wound healing. Toward an approximation of human chronic cutaneous ulcers in rabbit and rat. Methods Mol Med. 2003;78:55-65. doi:10.1385/1-59259-332-1:055

6. Nunan R, Harding KG, Martin P. Clinical challenges of chronic wounds: searching for an optimal animal model to recapitulate their complexity. Dis Model Mech. 2014;7(11):1205-13. doi: 10.1242/dmm.016782.

7. Sen CK, Gordillo GM, Roy S, Kirsner R, Lambert L, Hunt TK, et al. Human skin wounds: a major and snowballing threat to public health and the economy. Wound Repair Regen. 2009;17(6):763-71. doi: 10.1111/j.1524475X.2009.00543.x.

8. Stechmiller JK, Childress B, Cowan L. Arginine supplementation and wound healing. Nutr Clin Pract. 2005;20(1):52-61. doi: 10.1177/011542650502000152.

9. Lee RH, Efron D, Tantry U, Barbul A. Nitric oxide in the healing wound: a time-course study. J Surg Res. 2001;101(1):104-8. doi: 10.1006/jsre.2001.6261.

10. Silva JJ, Pompeu DG, Ximenes NC, Duarte AS, Gramosa NV, Carvalho Kde M, et al. Effects of Kaurenoic Acid and Arginine on Random Skin Flap Oxidative Stress, Inflammation, and Cytokines in Rats. Aesthetic Plast Surg. 2015;39(6):971-7. doi:10.1007/s00266-015-0559-8

11. Verma SK, Maheshwari S, Singh RK, Chaudhari PK. Laser in dentistry: An innovative tool in modern dental practice. Natl J Maxillofac Surg. 2012;3(2):124. doi: 10.4103/09755950.111342.

12. Farivar S, Malekshahabi T, Shiari R. Biological effects of low level laser therapy. J Lasers Med Sci. 2014;5(2):58.

13. Dungel P, Hartinger J, Chaudary S, Slezak P, Hofmann A, Hausner $\mathrm{T}$, et al. Low level light therapy by LED of different wavelength induces angiogenesis and improves ischemic wound healing. Lasers Surg Med. 2014 Dec;46(10):773-80. doi: 10.1002/lsm.22299.

14. Prado RP, Pinfildi CE, Liebano RE, Hochman BS, Ferreira LM. Effect of application site of low-level laser therapy in random cutaneous flap viability in rats. Photomed Laser Surg. 2009 Jun;27(3):411-6. doi: 10.1089/pho.2008.2320.

15. Ruh AC, Frigo L, Cavalcanti M, Svidnicki P, Vicari VN, Lopes-Martins RAB, et al. Laser photobiomodulation in pressure ulcer healing of human diabetic patients: gene expression analysis of inflammatory biochemical markers. 
Lasers Med Sci. 2018 Jan;33(1):165-71. doi: 10.1007/ s10103-017-2384-6.

16. Mostafavinia A, Bidram M, Gomi Avili A, Mahmanzar M, Karimifard SA, Sajadi E, et al. An improvement in acute wound healing in rats by the synergistic effect of photobiomodulation and arginine. Lab Anim Res. 2019;35:28. doi: 10.1186/s42826-019-0025-x.

17. Ebrahimpour-Malekshah R, Amini A, Zare F, Mostafavinia A, Davoody S, Deravi N, et al. Combined therapy of photobiomodulation and adipose-derived stem cells synergistically improve healing in an ischemic, infected and delayed healing wound model in rats with type 1 diabetes mellitus. BMJ Open Diabetes Res Care. 2020 Feb;8(1):e001033. doi:10.1136/bmjdrc-2019-001033.

18. Moradi A, Zare F, Mostafavinia A, Safaju S, Shahbazi A, Habibi M, et al. Photobiomodulation plus Adipose-derived Stem Cells Improve Healing of Ischemic Infected Wounds in Type 2 Diabetic Rats. Sci Rep. 2020 Jan 27;10(1):1206. doi: 10.1038/s41598-020-58099-z.

19. Moradi A, Kheirollahkhani Y, Fatahi P, Abdollahifar MA, Amini A, Naserzadeh P, et al. An improvement in acute wound healing in mice by the combined application of photobiomodulation and curcumin-loaded iron particles. Lasers Med Sci. 2019;34(4):779-791. doi: 10.1007/s10103018-2664-9.

20. Pouriran R, Piryaei A, Mostafavinia A, Zandpazandi S, Hendudari F, Amini A, et al. the effect of combined pulsed wave low-level laser therapy and human bone marrow mesenchymal stem cell-conditioned medium on open skin wound healing in diabetic rats. Photomed Laser Surg. 2016 Aug;34(8):345-54. doi: 10.1089/pho.2015.4020.

21. Barre-Sinoussi F, Montagutelli X. Animal models are essential to biological research: issues and perspectives. Future Sci OA. 2015 Nov;1(4):Fso63. doi: 10.4155/fso.15.63.

22. Salcido R, Popescu A, Ahn C. Animal models in pressure ulcer research. J Spinal Cord Med. 2007 Jan 1;30(2):107-16. doi: 10.1080/10790268.2007.11753921.

23. Armstrong DG, Meyr AJ. Risk factors for impaired wound healing and wound complications. UpToDate. Retrieved December. 2018;4:2018.

24. Naldaiz-Gastesi N, Goicoechea M, Alonso-Martin S, Aiastui A, Lopez-Mayorga M, Garcia-Belda P, et al. Identification and characterization of the dermal panniculus carnosus muscle stem cells. Stem Cell Reports. 2016 Sep 13;7(3):41124. doi: 10.1016/j.stemcr.2016.08.002.

25. Gould LJ, Leong M, Sonstein J, Wilson S. Optimization and validation of an ischemic wound model. Wound Repair Regen. 2005 Nov-Dec;13(6):576-82. doi: 10.1111/j.1524475X.2005.00080.x.

26. Chen C, Schultz GS, Bloch M, Edwards PD, Tebes S, Mast BA. Molecular and mechanistic validation of delayed healing rat wounds as a model for human chronic wounds. Wound Repair Regen. 1999 Nov-Dec;7(6):486-94. doi: 10.1046/j.1524-475X.1999.00486.x

27. Cooper DR, Wang C, Patel R, Trujillo A, Patel NA, Prather J, et al. Human Adipose-Derived Stem Cell Conditioned Media and Exosomes Containing MALAT1 Promote Human Dermal Fibroblast Migration and Ischemic Wound Healing. Adv Wound Care. 2018 Sep 1;7(9):299-308. doi: 10.1089/wound.2017.0775.
28. Fukui T, Kawaguchi AT, Takekoshi S, Miyasaka M, Sumiyoshi H, Tanaka R. Liposome-Encapsulated Hemoglobin Accelerates Skin Wound Healing in Diabetic dB/dB Mice. Artif Organs. 2017 Apr;41(4):319-26. doi: 10.1111/aor.12864.

29. Ren L, B Zhou, L Chen. Silicone ring implantation in an excisional murine wound model. Wounds, 2012. 24(2): 3642.

30. Frykberg RG, Banks J. Challenges in the Treatment of Chronic Wounds. Adv Wound Care. 2015 Sep 1;4(9):56082. doi: 10.1089/wound.2015.0635.

31. Velnar T, Bailey T, Smrkolj V. The wound healing process: an overview of the cellular and molecular mechanisms. $J$ Int Med Res. 2009 Sep-Oct;37(5):1528-42. doi: 10.1177/147323000903700531.

32. Franz MG, Kuhn MA, Wright TE, Wachtel TL, Robson MC. Use of the wound healing trajectory as an outcome determinant for acute wound healing. Wound Repair Regen. 2000 Nov-Dec;8(6):511-6. doi: 10.1046/j.1524475x.2000.00511.x.

33. Cirocchi R, Randolph J, Montedori A, et al. Staples versus sutures for surgical wound closure in adults. Cochrane Database Syst Rev. 2018;2018(5):CD011250. Published 2018 May 30. doi:10.1002/14651858.CD011250.pub2

34. de Freitas LF, Hamblin MR. Proposed Mechanisms of Photobiomodulation or Low-Level Light Therapy. IEEE J Sel Top Quantum Electron. 2016;22(3):7000417. doi: 10.1109/JSTQE.2016.2561201.

35. Carroll JD, Milward MR, Cooper PR, Hadis M, Palin WM. Developments in low level light therapy (LLLT) for dentistry. Dent Mater. 2014;30(5):465-75. doi: 10.1016/j. dental.2014.02.006.

36. Kouhkheil R, Fridoni M, Piryaei A, Taheri S, Chirani AS, Anarkooli IJ, et al. The effect of combined pulsed wave low-level laser therapy and mesenchymal stem cellconditioned medium on the healing of an infected wound with methicillin-resistant Staphylococcal aureus in diabetic rats. J Cell Biochem. 2018 Jul;119(7):5788-97. doi: 10.1002/ jcb.26759.

37. Fridoni M, Kouhkheil R, Abdollhifar MA, Amini A, Ghatrehsamani M, Ghoreishi SK, et al. Improvement in infected wound healing in type 1 diabetic rat by the synergistic effect of photobiomodulation therapy and conditioned medium. J Cell Biochem. 2019;120(6):9906-16. doi: $10.1002 / j \mathrm{jcb} .28273$.

38. Amir A, Solomon AS, Giler S, Cordoba M, Hauben DJ. The influence of helium-neon laser irradiation on the viability of skin flaps in the rat. Br J Plast Surg. 2000 Jan;53(1):58-62. doi: 10.1054/bjps.1999.3185.

39. Prado RP, Garcia SB, Thomazini JA, Piccinato CE. Effects of 830 and $670 \mathrm{~nm}$ laser on viability of random skin flap in rats. Photomed Laser Surg. 2012 Aug;30(8):418-24. doi: 10.1089/pho.2011.3042.

40. Prado R, Neves L, Marcolino A, Ribeiro T, Pinfildi C, Ferreira L, et al. Effect of low-level laser therapy on malondialdehyde concentration in random cutaneous flap viability. Photomed Laser Surg. 2010;28(3):379-84. doi: 10.1089/pho.2009.2535.

41. Cury V, Moretti AI, Assis L, Bossini P, Crusca Jde S, Neto $\mathrm{CB}$, et al. Low level laser therapy increases angiogenesis in 
a model of ischemic skin flap in rats mediated by VEGF, HIF-1alpha and MMP-2. J Photochem Photobiol B. 2013 Aug 5;125:164-70. doi: 10.1016/j.jphotobiol.2013.06.004.

42. Cury V, Bossini PS, Fangel R, Crusca Jde S, Renno AC, Parizotto NA. The effects of $660 \mathrm{~nm}$ and $780 \mathrm{~nm}$ laser irradiation on viability of random skin flap in rats. Photomed Laser Surg. 2009 Oct;27(5):721-4. doi: 10.1089/ pho.2008.2383.

43. Baldan CS, Masson IF, Esteves Junior I, Baldan AM, Machado AF, Casaroto RA, et al. Inhibitory effects of low-level laser therapy on skin-flap survival in a rat model. Plast Surg (Oakv). 2015;23(1):35-9. doi: 10.1177/229255031502300106.

44. Costa MS, Pinfildi CE, Gomes HC, Liebano RE, Arias VE, Silveira TS, et al. Effect of low-level laser therapy with output power of $30 \mathrm{~mW}$ and $60 \mathrm{~mW}$ in the viability of a random skin flap. Photomed Laser Surg. 2010 Feb;28(1):5761. doi: 10.1089/pho.2008.2444.

45. Alexander JW, Supp DM. Role of Arginine and Omega-3 Fatty Acids in Wound Healing and Infection. Adv Wound Care (New Rochelle). 2014 Nov 1;3(11):682-90. doi: 10.1089/wound.2013.0469.

46. Farreras N, Artigas V, Cardona D, Rius X, Trias M, Gonzalez JA. Effect of early postoperative enteral immunonutrition on wound healing in patients undergoing surgery for gastric cancer. Clin Nutr. 2005; 24(1):55-65. doi: 10.1016/j. clnu.2004.07.002.

47. Okamoto Y, Okano K, Izuishi K, Usuki H, Wakabayashi $\mathrm{H}$, Suzuki Y. Attenuation of the systemic inflammatory response and infectious complications after gastrectomy with preoperative oral arginine and omega- 3 fatty acids supplemented immunonutrition. World J Surg. 2009 Sep;33(9):1815-21. doi: 10.1007/s00268-009-0140-1.

48. Fazli M, Bjarnsholt T, Kirketerp-Møller K, Jørgensen B, Andersen AS, Krogfelt KA, Givskov M, Tolker-Nielsen T. Nonrandom distribution of Pseudomonas aeruginosa and Staphylococcus aureus in chronic wounds. J Clin Microbiol. 2009 Dec;47(12):4084-9. doi: 10.1128/JCM.01395-09.

49. Serra R, Grande R, Butrico L, et al. Chronic wound infections: the role of Pseudomonas aeruginosa and Staphylococcus aureus. Expert Rev Anti Infect Ther. 2015;13(5):605-613. doi:10.1586/14787210.2015.1023291 